\title{
Differences in gut microbiota composition between obese and lean children: a cross-sectional study
}

\author{
Liene Bervoets $^{1,2^{*}}$, Kim Van Hoorenbeeck ${ }^{3,4}$, Ineke Kortleven ${ }^{5}$, Caroline Van Noten ${ }^{5}$, Niel Hens ${ }^{5,6,7}$, Carl Vael ${ }^{7,8}$,
} Herman Goossens ${ }^{7}$, Kristine N Desager ${ }^{3,4}$ and Vanessa Vankerckhoven ${ }^{7}$

\begin{abstract}
Background: An altered gut microbiota composition has recently been linked to obesity. The principal aim of this study is to investigate and compare the gut microbiota composition in obese and lean children. Secondly, associations between analysed gut bacterial species, dietary compounds, energy intake and biochemical blood parameters are evaluated.

Methods: In this prospective cross-sectional study, 26 overweight/obese (mean BMI: $28.7 \pm 6.5$ ) and 27 lean (mean BMI: $16.5 \pm 2.1)$ children aged 6 to 16 were included. Faecal samples were collected and subjected to selective plating and quantitative real-time PCR (qPCR) in order to determine the concentrations of bacterial species belonging to the genera: Bacteroides, Bifidobacterium, Clostridium, Staphylococcus and Lactobacillus. Matrix-assisted laser desorption/ionization time-of-flight mass spectrometry (MALDI-TOF MS) was applied for an in-depth identification of species of Bacteroides fragilis group. Differences in the concentrations of gut bacterial species between obese and lean children were statistically analysed using Mann Whitney $U$ test. Subsequently, random forest analysis and multiple linear regression analysis were performed in order to test associations between gut bacterial species, dietary compounds and blood parameters.

Results: Obese children showed an elevated Firmicutes-to-Bacteroidetes ratio compared with lean children. Furthermore, low relative proportions of B. vulgatus and high concentrations of Lactobacillus spp. were observed in the obese microbiota. In all children, Staphylococcus spp. were positively associated with energy intake. Additionally, in obese children, Lactobacillus spp. were positively associated with plasma hs-CRP.
\end{abstract}

Conclusions: Our findings corroborate a significant difference in the gut microbiota composition of important bacterial species between obese and lean children. In future, non-invasive manipulation of gut microbiota composition in early infancy could offer a new approach to manage childhood obesity and associated disorders.

Keywords: Gut microbiota, 165 rDNA, MALDI-TOF MS, Bacteroides fragilis group, Obesity, Children

\section{Background}

Although there is evidence that the prevalence of childhood obesity is stabilising at different levels in different countries [1], the number of children and adolescents being overweight or obese is still dramatically high [2,3]. The major concern is that these children are at high risk

\footnotetext{
*Correspondence: liene.bervoets@uhasselt.be

${ }^{1}$ Faculty of Pharmaceutical, Biomedical and Veterinary Sciences, University of Antwerp, Antwerp, Belgium

${ }^{2}$ Faculty of Medicine and Life Sciences, Hasselt University, Agoralaan

1-Building D, 3590, Diepenbeek, Belgium

Full list of author information is available at the end of the article
}

of developing severe co-morbidities such as metabolic syndrome, non-alcohol fatty liver disease, type 2 diabetes mellitus and premature cardiovascular diseases $[4,5]$. Moreover, obese children are highly prone to become obese adults, especially when having a high body mass index (BMI) or an obese parent [6,7]. In order to combat childhood obesity and related complications, prevention is crucial. At the moment, the most important strategies to manage childhood obesity are therapeutic lifestyle changes, such as changing dietary habits and the physical activity level. However, these are often difficult to 
achieve. When lifestyle modifications continue to fail, pharmacological interventions and possibly bariatric surgery could be considered.

Nowadays, it is generally accepted that the development of obesity is caused by gene-environment interactions, generating a chronic positive energy balance [8]. However, physiological and environmental predispositions underlying obesity and associated metabolic disorders are still underexplored. Recent evidence suggests that our gut microbiota is involved in energy regulation as well as inflammation [9], and should therefore be considered as an environmental factor playing a role in the pathophysiology of obesity $[10,11]$. Although energy intake can affect the gut microbiota composition [12], it is still unclear whether the gut microbiota play a causal role in the development of obesity in humans.

So far, several studies in humans and mice have shown differences in gut microbiota composition between obese and lean subjects. These differences were mostly detected at the phylum level of mainly Firmicutes and Bacteroidetes [11-14]. Obesity in humans has already been associated with low intestinal concentrations of Bacteroidetes and high concentrations of Firmicutes, although this finding has been contradicted by other studies $[15,16]$. Only few studies have investigated the prevalence of faecal bacterial phyla in obese children and adolescents. One study demonstrated low concentrations of Bacteroidetes and high concentrations of Firmicutes in the distal gut of obese adolescents living in Spain [17]. Another study in Sweden, did not find significant differences in the concentrations of Bacteroides fragilis group, Lactobacillus spp. and Bifidobacterium spp. between preschool children with excessive body weight and normal-weight children [18]. By contrast, Vael et al. [19] demonstrated that a high intestinal concentration of Bacteroides fragilis group present in early infancy was associated with a higher risk of obesity later in life. In general, limited and contradictory findings with regard to the composition of the gut microbiota in obese children indicate that further in-depth analysis of the role of the intestinal microbiota in childhood obesity is warranted.

The principal aim of this study is to evaluate and compare the presence of certain gut bacterial species in faecal samples of obese and lean children and adolescents. Quantitative culturing was used to identify and determine the concentrations of the following bacterial genera: Bacteroides fragilis group, Bifidobacterium, Clostridium, Staphylococcus and Lactobacillus. In addition to quantitative culturing, matrix-assisted laser desorption/ ionization mass spectrometry (MALDI-TOF MS) was used for in-depth analysis of species belonging to the Bacteroides fragilis group. Quantitative real-time polymerase chain reaction (qPCR) was applied to quantify
Bacteroides-Prevotella-Porphyromonas spp., Bifidobacterium spp., Clostridium coccoides-Eubacterium rectale group, Clostridium leptum group, Staphylococcus spp. and Lactobacillus spp. The Firmicutes-to-Bacteroidetes ratio was calculated based on the qPCR results. Finally, analysed gut bacterial species were associated with dietary compounds and energy intake, which were assessed by dietary records. Moreover, concentrations of biochemical blood parameters were measured in overweight and obese subjects.

\section{Results}

\section{Subject characteristics}

Characteristics of the study population are shown in Table 1 . In total, 9 overweight, 7 obese, 10 morbidly obese $(\mathrm{O} / \mathrm{O})$ children and 21 normal-weight, 5 thinness grade I and 1 thinness grade III (C) children were included (see Methods section for details on BMI classification). Age, gender, height and dietary intake were not significantly different between the two study groups.

\section{Quantification of bacterial genera and species of Bacteroides fragilis group}

Differences in the concentrations of bacterial genera between $\mathrm{O} / \mathrm{O}$ and $\mathrm{C}$ children are presented in Figure 1A, B and $\mathrm{C}$. Figure $1 \mathrm{~A}$ illustrates differences between gut microbiota in $\mathrm{O} / \mathrm{O}$ and $\mathrm{C}$ children detected by quantitative plating. Bacteroides fragilis group and Clostridium spp. were borderline, but non-significantly different between $\mathrm{O} / \mathrm{O}$ and $\mathrm{C}$ children $(5.69 \pm 2.14$ vs. $6.66 \pm 0.84$ and $5.94 \pm 1.10$ vs. $6.31 \pm 0.80$, respectively; $\mathrm{p}=0.050$ and $\mathrm{p}=0.074)$. In-depth analysis of species belonging to the Bacteroides fragilis group using MALDI-TOF MS revealed dominating relative proportions of $B$. fragilis

Table 1 General characteristics of the studied population

\begin{tabular}{lccc}
\hline & $\mathbf{O} / \mathbf{O}(\mathbf{n}=\mathbf{2 6})$ & $\mathbf{C}(\mathbf{n}=\mathbf{2 7})$ & $\mathbf{p}$ value \\
\hline Anthropometric data & & & \\
\hline Gender (F/M), $\mathrm{n}$ & $12 / 14$ & $11 / 16$ & 0.691 \\
\hline Age, y & $11.64 \pm 2.43$ & $10.70 \pm 3.12$ & 0.229 \\
\hline Height, $\mathrm{cm}$ & $153.6 \pm 15.7$ & $144.7 \pm 18.6$ & 0.063 \\
\hline Weight, $\mathrm{kg}$ & $69.8 \pm 25.1$ & $35.7 \pm 12.6$ & $<0.0001^{*}$ \\
\hline BMl, $\mathrm{kg} / \mathrm{m}^{2}$ & $28.73 \pm 6.53$ & $16.48 \pm 2.10$ & $<0.0001^{*}$ \\
\hline BMl SDS & $2.69 \pm 0.80$ & $-0.43 \pm 0.96$ & $<0.0001^{*}$ \\
\hline
\end{tabular}

Dietary data

\begin{tabular}{lcccc}
\hline Number, $n$ & 22 & & 25 & \\
\hline Energy, kcal/d & $2231.95 \pm 437.69$ & & $082.32 \pm 446.79$ & 0.254 \\
\hline Carbohydrates, en\% & $45.68 \pm 9.55$ & & $48.81 \pm 6.28$ & 0.199 \\
\hline Fat, en\% & $40.08 \pm 9.73$ & & $38.59 \pm 6.01$ & 0.537 \\
\hline Protein, en\% & $14.43 \pm 3.94$ & & $14.48 \pm 6.54$ & 0.976 \\
\hline Dietary fibre, g/d & $19.26 \pm 8.33$ & & $15.64 \pm 7.33$ & 0.120 \\
\hline
\end{tabular}

O/O: obese group; C: control group; BMI SDS: body mass index standard deviation score. *Significant differences with $p$ value $<0.05$. 


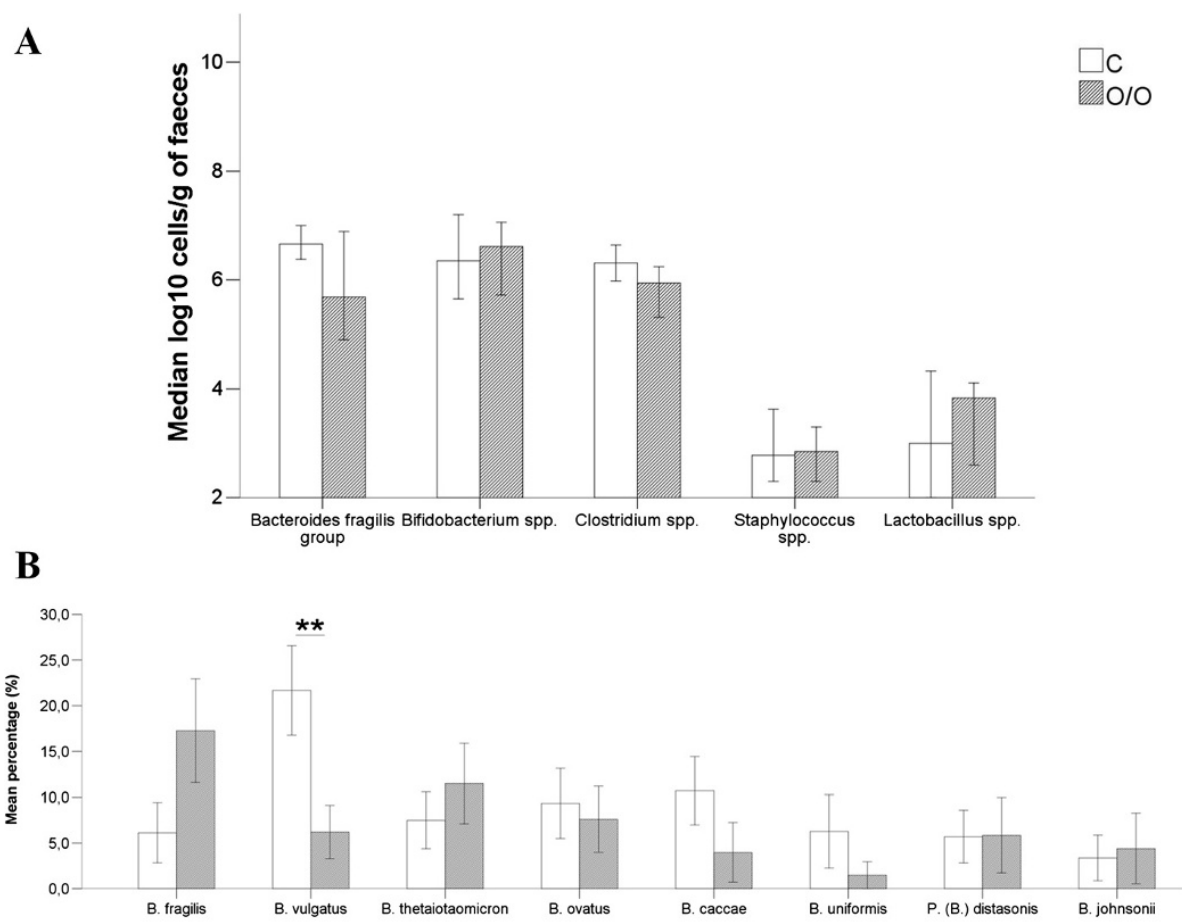

C

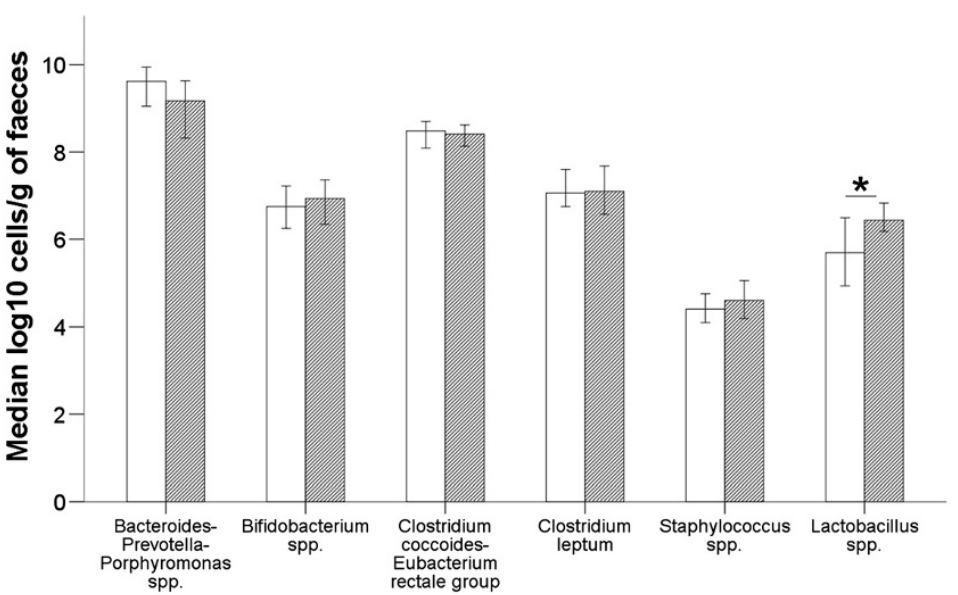

Figure 1 Differences in bacterial genera between $\mathrm{O} / \mathrm{O}$ and $\mathrm{C}$ group. A: Differences in bacterial genera between $\mathrm{O} / \mathrm{O}$ and $\mathrm{C}$ detected by quantitative plating. B: Differences in relative proportions of Bacteroides fragilis group species between O/O and C detected by MALDI-TOF MS. C Differences in bacterial genera between $\mathrm{O} / \mathrm{O}$ and $\mathrm{C}$ detected by $\mathrm{qPCR}$. Data of quantitative plating and $\mathrm{qPCR}$ are expressed as mean log ${ }_{10}$ cells/g of faeces. Data of MALDI-TOF MS are reported in percentages (\%). O/O: obese group; C: control group. Error bars $95 \% \mathrm{Cl}$. ${ }^{* *} \mathrm{p}=0.004 .{ }^{*} \mathrm{p}=0.04$.

$(17.3 \%$ vs. $6.1 \%, \mathrm{p}=0.136)$ and $B$. thetaiotaomicron $(11.5 \%$ vs. $7.5 \%, \mathrm{p}=0.930)$ in faecal samples of $\mathrm{O} / \mathrm{O}$ children compared with $C$ children (Figure 1B). By contrast, in $C$ children, relative proportions of B. caccae $(10.7 \%$ vs. $4.0 \%, \mathrm{p}=0.051), B$. ovatus $(9.3 \%$ vs. $7.6 \%, \mathrm{p}=0.585)$, B. uniformis $(6.3 \%$ vs. $1.5 \%, \mathrm{p}=0.177)$ and $B$. vulgatus $(21.7 \%$ vs. $6.2 \%, \mathrm{p}=0.004)$ prevailed. Note that only $B$. vulgatus proportions were significantly different between the $\mathrm{O} / \mathrm{O}$ and $\mathrm{C}$ children. Figure $1 \mathrm{C}$ shows differences found between gut bacterial species in $\mathrm{O} / \mathrm{O}$ and $\mathrm{C}$ children detected by qPCR. In contrast to the quantitative plating results, faecal concentrations of Lactobacillus spp. were found to be significantly higher in $\mathrm{O} / \mathrm{O}$ compared with $C$ children and adolescents $(6.44 \pm 1.20$ vs. $5.69 \pm 1.80, \mathrm{p}=0.035)$ using $\mathrm{qPCR}$.

\section{The Firmicutes-to-Bacteroidetes ratio}

In Figure 2, a boxplot of the Firmicutes-to-Bacteroidetes ratio of $\mathrm{O} / \mathrm{O}$ and $\mathrm{C}$ children is presented. The ratio resulted in favour of the Firmicutes in $\mathrm{O} / \mathrm{O}$ children and adolescents $(\mathrm{p}=0.007)$. 


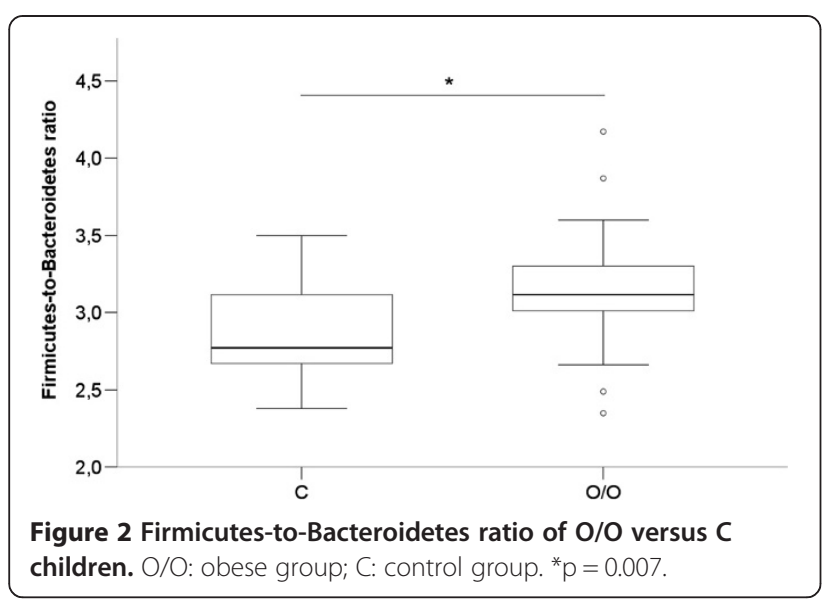

\section{Dietary assessment}

The most important associations between analysed gut bacterial species, dietary compounds and energy intake in a subsample of $22 \mathrm{O} / \mathrm{O}$ and $25 \mathrm{C}$ children are presented in Table 2. Children and adolescents with a high daily energy intake showed high faecal concentrations of Staphylococcus spp., analysed by means of quantitative plating $(\mathrm{p}=0.028)$.

\section{Biochemical markers}

Important biochemical markers were measured in fasting venous blood samples of 19 obese children (see Material and Method section for more details on blood sampling procedure). The following mean values were obtained: fasting plasma glucose: $82.44 \pm 5.34 \mathrm{mg} / \mathrm{dl}$; fasting plasma insulin: $21.58 \pm 17.31 \mu \mathrm{U} / \mathrm{ml}$; total cholesterol (TC): $176.26 \pm 42.14 \mathrm{mg} / \mathrm{dl}$; high-density lipoprotein (HDL) cholesterol: $49.61 \pm 10.05 \mathrm{mg} / \mathrm{dl}$; triglycerides (TG): $112.63 \pm 81.92 \mathrm{mg} / \mathrm{dl}$; leukocytes: $7.86 \pm 2.42 \%$; high-sensitive C-reactive protein (hs-CRP): $0.46 \pm 0.36$ $\mathrm{mg} / \mathrm{dl}$; alanin aminotransferase (ALT): $38.12 \pm 19.82 \mathrm{U} / \mathrm{l}$; aspartate aminotransferase (AST): $33.94 \pm 12.79 \mathrm{U} / \mathrm{l}$. The most important associations between major gut microbiota species and the concentration of the biochemical markers are presented in Table 3. Intestinal concentrations of Lactobacillus spp., which were analysed by quantitative plating, showed a positive association with plasma hs-CRP levels $(\mathrm{p}=0.007)$.

\section{Discussion}

At birth, the gut microbiota of an infant is sterile but rapidly assembles over days or months [20]. Mode of delivery (natural delivery versus caesarean section) and feeding method (breast feeding versus bottle feeding) have an early impact on the development of a child's gut microbiome [21]. At the age of four, the gut microbiota is fully mature [22]. Eventually, each person develops a unique gut microbiota which is stable over time in healthy adults [23].

In this cross-sectional study, the obese gut microbiota composition was compared with that of a lean one. We focused on two major phyla Bacteroidetes and Firmicutes, next to the Bacteroides fragilis group, Bifidobacterium, Clostridium, Staphylococcus and Lactobacillus. Different bacterial groups were selected according to the frequency to which they have been described in relevant literature $[17-19,24]$ and the ease of detection by the techniques used. On the one hand, quantitative plating was used as the 'gold standard' technique to isolate and characterise the selected bacterial groups. However, only 10 to $50 \%$ of all bacteria associated with the human body can be cultivated successfully $[23,25]$. Subsequently, high-throughput culture-independent techniques,

Table 2 Most important associations between gut microbiota and dietary compounds represented by regression coefficient $\beta$ ( $p$ value)

\begin{tabular}{|c|c|c|c|c|c|}
\hline & \multicolumn{5}{|c|}{ Dietary compounds } \\
\hline & Carbohydrates (en\%) & Fat (en\%) & Protein (en\%) & Fibre $(g / d)$ & Energy intake (kcal/d) \\
\hline \multicolumn{6}{|l|}{ Quantitative plating } \\
\hline Bacteroides fragilis group & $0.127(0.110)$ & $0.129(0.129)$ & $0.001(0.987)$ & & $-0.000(0.620)$ \\
\hline Bifidobacterium spp. & $-0.025(0.410)$ & & & & $0.000(0.822)$ \\
\hline Clostridium spp. & & & & & $0.001(0.156)$ \\
\hline Staphylococcus spp. & & & & & $0.001(0.028)^{*}$ \\
\hline Lactobacillus spp. & $0.069(0.360)$ & $0.104(0.180)$ & & & \\
\hline \multicolumn{6}{|l|}{ qPCR } \\
\hline Bacteroides-Prevotella-Porphyromonas spp. & $0.048(0.319)$ & $0.069(0.220)$ & & & $-0.000(0.830)$ \\
\hline Bifidobacterium spp. & $0.017(0.439)$ & & & & $0.000(0.285)$ \\
\hline Clostridium coccoides-Eubacterium rectale group & & & & & $0.000(0.269)$ \\
\hline Clostridium leptum group & & & & & $0.000(0.240)$ \\
\hline Staphylococcus spp. & $-0.093(0.203)$ & $-0.084(0.285)$ & $-0.083(0.075)$ & & $0.000(0.691)$ \\
\hline Lactobacillus spp. & $0.052(0.503)$ & $0.069(0.401)$ & $-0.000(0.993)$ & $0.010(0.745)$ & $0.000(0.415)$ \\
\hline
\end{tabular}

Regression coefficient with $95 \% \mathrm{Cl}$. ${ }^{*}$ Significant association with $\mathrm{p}$ value $<0.05$. 
Table 3 Most important associations between biochemical parameters, gut microbiota and BMI SDS represented by regression coefficient $\beta$ ( $p$ value)

\begin{tabular}{|c|c|c|c|c|c|c|c|c|c|}
\hline & \multicolumn{9}{|c|}{ Biochemical markers } \\
\hline & $\begin{array}{c}\text { Leukocytes } \\
\text { (\%) }\end{array}$ & $\begin{array}{l}\text { Glucose } \\
\text { (mg/dl) }\end{array}$ & $\begin{array}{l}\text { Insulin } \\
(\mu \mathrm{U} / \mathrm{ml})\end{array}$ & $\begin{array}{l}\text { HDL-C } \\
\text { (mg/dl) }\end{array}$ & $\begin{array}{c}\text { TG } \\
(\mathrm{mg} / \mathrm{dl})\end{array}$ & $\begin{array}{c}\mathrm{TC} \\
(\mathrm{mg} / \mathrm{dl})\end{array}$ & $\begin{array}{l}\text { ALT } \\
(U / I)\end{array}$ & $\begin{array}{l}\text { AST } \\
(\mathrm{U} / \mathrm{l})\end{array}$ & $\begin{array}{r}\text { hs-CRP } \\
\text { (mg/l) }\end{array}$ \\
\hline \multicolumn{10}{|l|}{ Quantitative plating } \\
\hline \multirow[t]{2}{*}{ Bacteroides fragilis group } & & -1.469 & & -1.677 & & -6.926 & & & \\
\hline & & $(0.237)$ & & $(0.099)$ & & $(0.497)$ & & & \\
\hline \multirow[t]{2}{*}{ Bifidobacterium spp. } & & 0.830 & & & & 5.673 & -1.619 & -0.124 & \\
\hline & & $(0.631)$ & & & & $(0.719)$ & $(0.753)$ & $(0.969)$ & \\
\hline \multirow[t]{2}{*}{ Clostridium spp. } & -0.276 & 0.071 & -3.725 & & 2.322 & 22.353 & & -3.598 & \\
\hline & $(0.562)$ & $(0.967)$ & $(0.343)$ & & $(0.932)$ & $(0.181)$ & & $(0.245)$ & \\
\hline \multirow[t]{2}{*}{ Staphylococcus spp. } & & & & 1.718 & -30.933 & -32.137 & & & \\
\hline & & & & $(0.414)$ & $(0.381)$ & $(0.122)$ & & & \\
\hline \multirow[t]{2}{*}{ Lactobacillus spp. } & & & 2.040 & & & & & & 0.171 \\
\hline & & & $(0.497)$ & & & & & & $(0.007)^{*}$ \\
\hline \multicolumn{10}{|l|}{ qPCR } \\
\hline \multirow[t]{2}{*}{ Bacteroides-Prevotella-Porphyromonas spp. } & & & & -0.668 & & & & & \\
\hline & & & & $(0.771)$ & & & & & \\
\hline \multicolumn{10}{|l|}{ Bifidobacterium spp. } \\
\hline \multirow[t]{2}{*}{ Clostridium coccoides-Eubacterium rectale group } & 0.860 & 0.825 & & 1.259 & & 33.654 & -3.686 & -6.465 & 0.285 \\
\hline & $(0.107)$ & $(0.810)$ & & $(0.688)$ & & $(0.234)$ & $(0.518)$ & $(0.189)$ & $(0.292)$ \\
\hline \multicolumn{10}{|l|}{ Clostridium leptum group } \\
\hline \multirow[t]{2}{*}{ Staphylococcus spp. } & & & & & 11.856 & & & & \\
\hline & & & & & $(0.481)$ & & & & \\
\hline \multirow[t]{2}{*}{ Lactobacillus spp. } & & -1.307 & 1.342 & & 17.604 & -5.764 & & 6.274 & -0.004 \\
\hline & & $(0.518)$ & $(0.703)$ & & $(0.468)$ & $(0.717)$ & & $(0.139)$ & $(0.979)$ \\
\hline \multirow[t]{2}{*}{ BMI SDS } & 0.726 & 2.449 & 1.008 & 2.588 & 75.925 & 29.824 & 19.310 & 10.655 & 0.181 \\
\hline & $(0.469)$ & $(0.392)$ & $(0.900)$ & $(0.406)$ & $(0.086)$ & $(0.207)$ & $(0.072)$ & $(0.117)$ & $(0.390)$ \\
\hline
\end{tabular}

Regression coefficient with $95 \% \mathrm{Cl}$. ${ }^{*}$ Significant association with $\mathrm{p}$ value $<0.05$.

which use DNA sequences encoding the $16 \mathrm{~S}$ ribosomal RNA subunit, were applied in order to assign an organism to a phylogenetic classification more accurately [25].

To our knowledge, our study was the first to perform an in-depth analysis of species belonging to the Bacteroides fragilis group by means of MALDI-TOF MS. Overall, our results reveal a high Firmicutes-to-Bacteroidetes ratio in faeces of obese children including alterations at species level.

Selective media have been used successfully to identify and enumerate Bacteroides fragilis group from human faeces [26]. For the first time, a further in-depth analysis of species of the Bacteroides fragilis group revealed reduced relative proportions of $B$. vulgatus in obese children and adolescents. One study reported decreased relative proportions of $B$. vulgatus in the faeces of type 2 diabetic subjects using species specific PCR-denaturing gradient gel electrophoresis (DGGE) [27]. B. vulgatus was found to constitute a part of the core gut microbiota in healthy humans and is generally considered to be a beneficial gut commensal [28]. These findings point towards a possible role for B. vulgatus in the pathophysiology of Western diseases, such as obesity and diabetes.

Moreover, the qPCR method that was used in this study to detect and quantify Bacteroidetes (BacteroidesPrevotella-Porphyromonas spp.), Firmicutes (Clostridium coccoides-Eubacterium rectale group, Clostridium leptum group, Staphylococcus spp. and Lactobacillus spp.), and Bifidobacterium spp. in human faeces has already been thoroughly evaluated and validated [29-31]. In agreement with the findings of previous studies [32,33], we describe higher concentrations of Lactobacillus spp. in the obese gut microbiota. However, the use of quantitative plating did not permit the detection of a significantly higher concentration of Lactobacillus spp. in faeces of obese children, which we did see using qPCR. A possible explanation is that L. gasseri and L. acidophilus could not be identified in culture due to the presence of vancomycin in the LAMVAB medium [34]. Nevertheless, both quantitative culturing and qPCR resulted in a similar proportion of Lactobacillus spp. in the obese gut microbiota. A study conducted by Million 
et al. [32], demonstrated that Lactobacillus reuteri was associated with obesity in adults. By contrast, Santacruz et al. [33] showed that BMI SDS reduction in obese adolescents led to a concomitant increase in the concentrations of Lactobacillus spp. These findings thus suggest a possible role of Lactobacillus at species level in body weight and obesity. Additionally, we showed that the concentration of Lactobacillus spp. is positively correlated to plasma hs-CRP levels in obese children and adolescents. An increased prevalence of positive Firmicutes to higher levels of plasma hs-CRP was also seen in a study conducted in 51 obese and 28 normal-weight children and adults [35]. These results seems therefore to suggest a possible role for Lactobacillus spp. in "lowgrade" inflammation, a major pathophysiological process of obesity.

Interestingly, we detected an elevated Firmicutes-to -Bacteroidetes ratio in the gut microbiota of obese children and adolescents. Previous investigators also showed significant associations between this ratio and obesity in mice and humans [11-14]. The results of our study are similar to a study in Spanish children, demonstrating increased concentrations of Firmicutes and decreased concentrations of Bacteroidetes in the obese gut [17]. Contrary to these findings, other studies described no or even opposite differences in the Firmicutes-to-Bacteroidetes ratio between obese and lean subjects $[15,16]$. Possibly, these variations in study outcome are related to the fact that different methodologies were applied in these studies.

To further elucidate the complex role of gut microbiota in host physiology, a more thorough examination of the influence of diet on gut microbiota is recommended. In order to do so, we analysed the relationship between the presence of certain gut bacterial species with dietary compounds and energy intake. Here, we demonstrate that, independent of the BMI status, children and adolescents with a high energy intake (expressed in $\mathrm{kcal} / \mathrm{d}$ ) possess high faecal concentrations of Staphylococcus spp. analysed by quantitative culture. Note that the regression coefficient $\beta$ of energy intake is low in all cases. This is due to the fact that values of energy intake are expressed in $\mathrm{kcal} / \mathrm{d}$. Given the range of energy intake (1635.53 to $2669.64 \mathrm{kcal})$, results in effect on mean concentration of Staphylococcus spp. of 1.27 to 2.08 are obtained. These results are not negligible and a real significant association has been detected. However, caution must be taken when translating these findings into a biological meaningful interpretation. Hence, more detailed research on this topic is necessary. Nevertheless, the importance of Staphylococcus spp. in childhood obesity has already been demonstrated by Kalliomaki et al. [24] who showed that a greater faecal concentration of Staphylococcus spp. during infancy predicted the development of overweight during childhood. A possible role of Staphylococcus spp. in energy harvesting during childhood is thus suggested.

One major limitation of the current study is the small sample size and therefore these results should be interpreted with caution. In addition, pregnancy related factors, social status, and the period of being obese prior to inclusion were not taken into account.

Further longitudinal research on the cause-effect relationship between gut microbiota and obesity is highly justified, since different bacterial species could play a significant role in the human energy harvest and weight regulation. Moreover, consideration of lifestyle factors in gut microbiota studies is highly recommended, since changes in dietary pattern and physical activity could influence gut microbiota composition and the development of obesity. Finally, we suggest to focus future research not only on the elucidation of gut microbiota composition in obese subjects, but also on the study of gut metabolites, i.e. "metabolomics". This suggestion for future research aims at expanding our knowledge on the complex interplay between gut microbiota, energy homeostasis and obesity.

In the future, modification of the gut microbiota composition by the administration of pro-, pre- or synbiotics in early childhood could offer an opportunity to prevent and/or treat obesity [36]. However, additional research is required.

\section{Conclusions}

In this study, important compositional differences in the faecal gut microbiota of obese and lean children were revealed. This was generally reflected by an elevated Firmicutes-to-Bacteroidetes ratio in the obese study population. At the species level, low proportions of $B$. vulgatus and high concentrations of Lactobacillus spp. were found in faeces of obese children and adolescents. Furthermore, the presence of Lactobacillus spp. in the obese gut microbiota was positively associated with plasma hs-CRP levels. We also found a positive association between energy intake and the presence of Staphylococcus spp. in faeces of children, independent of their BMI status. The aforementioned bacterial genera and species may thus be more efficient at extracting energy from a given diet in obese children and adolescents compared with gut microbiota of lean children. Hence, B. fragilis group, Lactobacillus spp. and Staphylococcus spp. play an important role in the pathophysiology of childhood obesity. We hypothesize that an aberrant gut microbiota composition in combination with influences of lifestyle factors might contribute to the development of childhood obesity. To further confirm this hypothesis, additional research is required in a longitudinal setting with recruitment of a larger sample population. Finally, prevention and treatment strategies based on modification 
of the gut microbiota in obese children might conceivably contribute to limit the development of future obesity.

\section{Methods}

\section{Subject characteristics}

Overweight, obese and morbidly obese (O/O: obese group) children were recruited from the paediatric obesity clinic at the Antwerp University Hospital. Normalweight and lean children ( $\mathrm{C}$ : control group) were recruited among the offspring of personnel working at the University of Antwerp. Skilled personnel recorded weight (scale, SECA 701 scale, Hamburg, Germany) and height (stadiometer, SECA 225, Hamburg, Germany) measurements, to the nearest $0.1 \mathrm{~kg}$ for weight and 1 $\mathrm{mm}$ for height and the BMI (in $\mathrm{kg} / \mathrm{m}^{2}$ ). All subjects were classified based on the international BMI cut-off values of the Extended International Obesity Task Force (IOTF) for children aged 2 to 18 [37]. These values are based on age and gender-specific centile curves passing through BMI 35 (morbid obesity), 30 (obesity), 25 (overweight) and 18.5, 17 and 16 (thinness grades I, II and III) at age 18. BMI standard deviation score (SDS) was assessed using an electronic calculator (Auxology 1.1, Pfizer, New York, USA) based on the local reference standards, i.e. Flemish growth charts [38]. Exclusion criteria included the use of corticosteroids or antibiotics in the month prior to the study as well as during the study and significant co-morbidities such as an acute infection, prematurity or chronic diseases. The study was conducted in accordance with the ethical rules of the Helsinki Declaration. Informed consent was obtained from all children and their parents or legal guardian. The study protocol was approved by the local medical Ethics Committee of the Antwerp University Hospital (document 7/41/226).

\section{Gut microbiota analyses \\ Faecal sample collection}

A fresh faecal sample was self-collected and stored immediately at $-20^{\circ} \mathrm{C}$. The collection took place in the same week as the dietary record. Subsequently, the faecal samples were transported to the laboratory and stored at $-80^{\circ} \mathrm{C}$ until further analysis.

\section{Quantitative plating}

Approximately $0.5 \mathrm{~g}$ of wet faeces was diluted in 9 volumes of phosphate buffer saline (PBS) and homogenized using a stomacher (Minimix ${ }^{\circ}$, Interscience, Arpents, France). Serial dilutions were plated using the Eddy Jet ${ }^{\circ}$ apparatus (Led Techno, Heusden-Zolder, Belgium) onto the selective growth media. More specifically, Staphylococcus spp. were cultivated on mannitol salt agar (MSA) (Becton-Dickinson, Erembodegem, Belgium); only yellow and white colonies were considered [39]. Bacteroides fragilis group was determined on Bacteroides Bile Esculin agar (BBE) (Becton-Dickinson, Erembodegem, Belgium); only black pigmented colonies were considered [40]. Clostridium spp. counts were obtained after pre-treatment of the faecal sample in 70\% ethanol for 30 minutes and subsequent culture on Columbia blood agar (CBA) [41]. Lactobacillus spp. were cultured on Lactobacillus anaerobic de Man, Rogosa and Sharpe with vancomycin and bromocresolgreen (LAMVAB) medium; only green and white colonies were considered [34]. Bifidobacterium spp. were determined on modified trypticase-phytone-yeast agar (MTPY); only colonies smaller than $0.7 \mathrm{~mm}$ were considered [42]. To confirm the presence of Bifidobacterium spp. in these colonies, a gram stain was conducted. The numbers of colonies positive for gram stain were expressed relative to the total number of colonies examined.

\section{MALDI-TOF MS analysis}

Of each sample, ten presumed colonies of Bacteroides fragilis group were subcultured on Wilkins-Chalgren medium for 24h and spotted onto a target plate. Matrixassisted laser desorption/ionization mass spectrometry (MALDI-TOF MS) analysis was accomplished according to the manufacturer's instructions (Microflex ${ }^{\text {Tx }}$ LT Bruker Daltonik GmbH, Bremen, Germany). Recorded mass spectra generated with the MALDI Biotyper 2.0 software package were compared with each entry of the MALDI Biotyper database and relationships were identified. According to the criteria by Nagy et al. [26], only identifications with a $\log$ (score) between 1.9 and 3.0 were considered to be significant and allowed for identification of the bacterial populations at the species level.

\section{Quantitative real-time $P C R$}

DNA extraction An aliquot $(200 \mu \mathrm{l})$ of faecal suspension was suspended in $300 \mu \mathrm{l}$ Tris-SDS solvent, $300 \mu \mathrm{g}$ glass beads (diameter, $0.1 \mathrm{~mm}$ ) and $500 \mu \mathrm{l}$ TE-saturated phenol. Further extraction of total bacterial DNA was performed as described by Matsuki et al. [30]. The mixture was vortexed vigorously with FastPrep ${ }^{\circ}$ FP120A (BIO $101^{\circ}$ Systems, Thermo Scientific) for $30 \mathrm{~s}$ at a power level of 5.0. After centrifugation for $5 \mathrm{~min}$ at 13 $000 \mathrm{rpm}, 400 \mu \mathrm{l}$ of the supernatant was collected and $400 \mu \mathrm{l}$ phenol/chloroform/isoamylalcohol (25:24:1) was added. Subsequently, $250 \mu \mathrm{l}$ of the supernatant was subjected to isopropanol.

qPCR qPCR assay was performed as previously described by Rinttilä et al. [31]. All PCR experiments were carried out in triplicate with a reaction volume of $25 \mu \mathrm{l}$. The reaction mixture contained $7.5 \mu \mathrm{l}$ sterile Milli-Q; $12.5 \mu \mathrm{l}$ iQ SYBR Green Supermix and $1.25 \mu \mathrm{l}$ of $10 \mu \mathrm{M}$ of each primer (forward and reverse). Subsequently, $2.5 \mu \mathrm{l}$ of faecal DNA was added to the reaction mixture. 
For each bacterial strain, positive controls were added to each reaction and the total bacterial copy number per organism was determined with $16 \mathrm{~S}$ rRNA gene targeted primers [29-31]. The bacterial primer sequences are reported in Table 4.

The Firmicutes-to-Bacteroidetes ratio An estimation of the total amount of Firmicutes was obtained by adding bacterial values (in $\log _{10}$ cells/g faeces) from Clostridium coccoides-Eubacterium rectale group (clostridial cluster XIV).

Clostridium leptum group (clostridial cluster IV), Lactobacillus spp. and Staphylococcus spp. For the total amount of Bacteroidetes, the Bacteroides-PrevotellaPorphyromonas spp. were taken into consideration. The ratio was calculated according to Mariat et al. [43].

\section{Dietary assessment}

A total of 22 (84.6\%) O/O and 25 (92.6\%) C children fully completed a five-day dietary record (three weekdays and two weekend days). The average daily intake of energy and nutrients was calculated with the use of Becel Institute Nutritional Software program. The dietary variables examined in this study were carbohydrates (energy \%), fat (energy \%), protein (energy \%), fibres (g/day), and total energy intake (kcal/day).

\section{Biochemical analyses}

Blood sampling was initially conducted to detect associated metabolic and inflammatory complications in the O/O group. Since only 19 out of 26 fasting venous blood samples of obese children were available, results of only these samples were considered. Glucose, total cholesterol
(TC), high-density lipoprotein cholesterol (HDL-C), triglycerides (TG), alanine aminotransferase (ALT), aspartate aminotransferase (AST) and high-sensitive C-reactive protein (hs-CRP) were measured on Dimension Vista 1500 System (Siemens Healthcare Diagnostics Inc., Neward, Delaware, USA). Insulin levels were measured using chemoluminescence (Roche Diagnostics, Rotkreuz, Switzerland). White blood cell count was performed using flow cytometry (Advia 2120, Siemens Healthcare Diagnostics Inc., Neward, Delaware, USA).

\section{Statistical analysis}

Descriptive and comparative analyses were performed in IBM SPSS version 20.0 (SPSS Inc., Chicago, IL, USA). The distribution of the residuals was tested for normality using the Kolmogorov-Smirnov test with Lilliefors correction. Independent samples $t$ test was used in case of no significant deviation from normality. Otherwise the Mann Whitney $U$ test was used. Chi square $\left(\chi^{2}\right)$ test of association was used to compare characteristics between the $\mathrm{O} / \mathrm{O}$ and $\mathrm{C}$ study groups. Data were presented as mean with standard deviation (mean \pm SD) unless indicated otherwise. Since bacterial counts followed a right-skewed distribution, data were $\log _{10}$-transformed. Bacterial data were expressed as median $\log _{10}$ cells/g of faeces with interquartile ranges (IQR). Regression analyses were implemented in $\mathrm{R}$ 2.13.1. Explanatory variables were selected on the basis of a random forest analysis, i.e. a nonparametric technique that facilitates the selection of the important variables in a regression setting [44]. Given the random forest variable importance plot, the most important predictors above a visually selected cut-off value were chosen. Subsequently,

Table 4 16S rRNA gene-targeted group specific primers per bacterial group/species used in this study

\begin{tabular}{|c|c|c|c|c|}
\hline Target & Primer sequence $\left(5^{\prime}-3^{\prime}\right)$ & Reference strains & $\begin{array}{l}\text { Annealing } \\
\text { temp }\left({ }^{\circ} \mathrm{C}\right)\end{array}$ & Reference \\
\hline \multicolumn{5}{|l|}{ Bacteroidetes } \\
\hline \multirow[t]{2}{*}{ Bacteroides-Prevotella-Porphyromonas spp. } & GGTGTCGGCTTAAGTGCCAT & Bacteroides fragilis DSM 2151/LMG 10263 & 68 & [31] \\
\hline & CGGA(C/T)GTAAGGGCCGTGC & & & \\
\hline \multicolumn{5}{|l|}{ Firmicutes } \\
\hline \multirow[t]{2}{*}{ Staphylococcus spp. } & GCGATTGATGGTGATACG & Staphylococcus aureus ATCC 29213 & 55 & [29] \\
\hline & AGCCAAGCCTTFACGAACTAAAGC & & & \\
\hline \multirow{2}{*}{$\begin{array}{l}\text { Clostridium coccoides-Eubacterium rectale } \\
\text { group (clostridial cluster XIV) }\end{array}$} & CGGTACCTGACTAAGAAGC & Ruminococcus productus DSM 2950/YIT 6141 & 55 & [31] \\
\hline & AGTT(C/T)ATTCTTGCGAACG & & & \\
\hline \multirow[t]{2}{*}{ C. leptum group (clostridial cluster IV) } & GCACAAGCAGTGGAGT & Faecalibacterium prausnitzii YIT 6174 & 50 & {$[30]$} \\
\hline & TTCCTCCGTTTTGTCAA & & & \\
\hline \multirow[t]{2}{*}{ Lactobacillus spp. } & AGCAGTAGGGAATCTTCCA & Lactobacillus acidophilus LMG 9433 & 58 & [31] \\
\hline & CACCGCTACACATGGAG & & & \\
\hline \multicolumn{5}{|l|}{ Actinobacteria } \\
\hline \multirow[t]{2}{*}{ Bifidobacterium spp. } & TCGCGTC(C/T)GGTGTGAAAG & Bifidobacterium longum DSM 20219/YIT 4021 & 58 & [31] \\
\hline & CCACATCCAGC(A/G)TCCAC & & & \\
\hline
\end{tabular}


multiple linear regression was applied to quantify the associations between gut microbiota, diet, biochemical parameters and BMI SDS controlled for age and gender. Wilcoxon-signed rank test was used to evaluate the difference of accuracy between quantitative culturing and qPCR. Statistical significance was assessed at the 5\% level.

\section{Abbreviations \\ ALT: Alanin aminotransferase; AST: Aspartate aminotransferase; BBE: Bacteroides Bile Esculin; BMI SDS: Body Mass Index Standard Deviation Score; C: Control group; CBA: Columbia Blood Agar; HDL: High-density lipoprotein; hs-CRP: High sensitive C-reactive protein; IOTF: International Obesity Task Force; IQR: Inter Quartile Ranges; LAMVAB: Lactobacillus Anaerobic de Man Rogosa and Sharpe with Vancomycin And Bromocresolgreen; MALDI-TOF MS: Matrix-Assisted Laser-Desorption /lonization Time-Of-Flight Mass Spectrometry; MSA: Mannitol Salt Agar; MTPY: Modified Trypticase-Phytone-Yeast Agar; O/O: Obese group; PBS: Phosphate Buffer Saline; TC: Total cholesterol; TG: Triglycerides; QPCR: Quantitative real-time polymerase chain reaction.}

\section{Competing interests}

All authors declare that there are no competing financial interests in relation to the work described.

\section{Authors' contributions}

$\mathrm{KD}, \mathrm{CV}, \mathrm{HG}$ and WK conceived and designed the study. LB, KVH, IK and $\mathrm{CVN}$ collected the data. LB carried out experiments. LB, IK, CVN and NH statistically analysed the data. $\mathrm{LB}, \mathrm{KVH}, \mathrm{NH}$ and $\mathrm{WK}$ interpreted the data. $\mathrm{LB}$, IK and CVN did literature research. LB generated figures and tables. LB wrote the manuscript with help of $\mathrm{KVH}$ and $\mathrm{WK}$. KVH, NH, KD, CV, HG and WK revised the paper. LB had full access to all of the data in the study and takes responsibility for the integrity of the data and accuracy of the data analysis. All authors had final approval of the submitted and published versions.

\section{Acknowledgements}

We thank Christine Lammens and Sabine Chapelle for their technical assistance in analysing the gut microbiota. We thank Racheli Banach and Annemie Van de Sompel for their help with the analysis of the dietary records. We thank dr. Eric Caers for the language editing of the manuscript. This study was funded by the Laboratory of Medical Microbiology, the Department of Pediatrics of the University of Antwerp, the Antwerp University Hospital and the foundation Limburg Sterk Merk, Hasselt University, Ziekenhuis Oost-Limburg and Jessa Hospital.

\section{Author details}

'Faculty of Pharmaceutical, Biomedical and Veterinary Sciences, University of Antwerp, Antwerp, Belgium. ${ }^{2}$ Faculty of Medicine and Life Sciences, Hasselt University, Agoralaan 1-Building D, 3590, Diepenbeek, Belgium. ${ }^{3}$ Laboratory of Experimental Medicine and Pediatrics, University of Antwerp, Antwerp, Belgium. ${ }^{4}$ Department of Pediatrics, Antwerp University Hospital, Antwerp, Belgium. ${ }^{5}$ Faculty of Medicine and Health Sciences, University of Antwerp, Antwerp, Belgium. ${ }^{6}$ Center for Statistics, Hasselt University, Diepenbeek, Belgium. 'Vaccine \& Infectious Disease Institute, University of Antwerp, Antwerp, Belgium. ${ }^{8}$ Department of Microbiology, Klina Hospital, Antwerp, Belgium.

Received: 18 March 2013 Accepted: 23 April 2013 Published: 30 April 2013

\section{References}

1. Olds T, Maher C, Zumin S, Peneau S, Lioret S, Castetbon K, et al: Evidence that the prevalence of childhood overweight is plateauing: data from nine countries. Int I Pediatr Obes 2011, 6:342-360.

2. Gupta N, Goel K, Shah P, Misra A: Childhood obesity in developing countries: epidemiology, determinants, and prevention. Endocr Rev 2012 33:48-70.

3. Lakshman R, Elks CE, Ong KK: Childhood obesity. Circulation 2012, 126:1770-1779.
4. Weiss R: Metabolic syndrome in childhood - causes and effects. Endocr Dev 2010, 19:62-72.

5. Burgert TS, Taksali SE, Dziura J, Goodman TR, Yeckel CW, Papademetris X, et al: Alanine aminotransferase levels and fatty liver in childhood obesity: associations with insulin resistance, adiponectin, and visceral fat. J Clin Endocrinol Metab 2006, 91:4287-4294.

6. Whitaker RC, Wright JA, Pepe MS, Seidel KD, Dietz WH: Predicting obesity in young adulthood from childhood and parental obesity. N Engl J Med 1997, 337:869-873.

7. Freedman DS, Khan LK, Serdula MK, Dietz WH, Srinivasan SR, Berenson GS: The relation of childhood BMI to adult adiposity: the Bogalusa Heart Study. Pediatrics 2005, 115:22-27.

8. Maffeis C: Aetiology of overweight and obesity in children and adolescents. Eur J Pediatr 2000, 159(Suppl 1):S35-S44.

9. Cani PD, Bibiloni R, Knauf C, Waget A, Neyrinck AM, Delzenne NM, et al: Changes in gut microbiota control metabolic endotoxemia-induced inflammation in high-fat diet-induced obesity and diabetes in mice. Diabetes 2008, 57:1470-1481.

10. Backhed F, Ding H, Wang T, Hooper LV, Koh GY, Nagy A, et al: The gut microbiota as an environmental factor that regulates fat storage. Proc Natl Acad Sci U S A 2004, 101:15718-15723.

11. Turnbaugh PJ, Ley RE, Mahowald MA, Magrini V, Mardis ER, Gordon Jl: An obesity-associated gut microbiome with increased capacity for energy harvest. Nature 2006, 444:1027-1031.

12. Murphy EF, Cotter PD, Healy S, Marques TM, O'Sullivan O, Fouhy F, et al: Composition and energy harvesting capacity of the gut microbiota: relationship to diet, obesity and time in mouse models. Gut 2010, 59:1635-1642

13. Ley RE, Backhed F, Turnbaugh P, Lozupone CA, Knight RD, Gordon Jl: Obesity alters gut microbial ecology. Proc Natl Acad Sci U S A 2005, 102:11070-11075.

14. Ley RE, Turnbaugh PJ, Klein S, Gordon J:: Microbial ecology: human gut microbes associated with obesity. Nature 2006, 444:1022-1023.

15. Duncan SH, Lobley GE, Holtrop G, Ince J, Johnstone AM, Louis P, et al: Human colonic microbiota associated with diet, obesity and weight loss. Int J Obes (Lond) 2008, 32:1720-1724.

16. Schwiertz A, Taras D, Schafer K, Beijer S, Bos NA, Donus C, et al: Microbiota and SCFA in lean and overweight healthy subjects. Obesity (Silver Spring) 2010, 18:190-195.

17. Ferrer M, Ruiz A, Lanza F, Haange SB, Oberbach A, Till H, et al: Microbiota from the distal guts of lean and obese adolescents exhibit partial functional redundancy besides clear differences in community structure. Environ Microbiol 2013, 15:211-226

18. Karlsson CL, Onnerfalt J, Xu J, Molin G, Ahrne S, Thorngren-Jerneck K: The microbiota of the gut in preschool children with normal and excessive body weight. Obesity (Silver Spring) 2012, 20:2257-2261.

19. Vael C, Verhulst SL, Nelen V, Goossens H, Desager KN: Intestinal microflora and body mass index during the first three years of life: an observational study. Gut Pathog 2011, 3:8

20. Mackie RI, Sghir A, Gaskins HR: Developmental microbial ecology of the neonatal gastrointestinal tract. Am J Clin Nutr 1999, 69:1035S-1045S.

21. Penders J, Thijs C, Vink C, Stelma FF, Snijders B, Kummeling I, et al: Factors influencing the composition of the intestinal microbiota in early infancy. Pediatrics 2006, 118:511-521.

22. Vrieze A, Holleman F, Zoetendal EG, de Vos WM, Hoekstra JB, Nieuwdorp M: The environment within: how gut microbiota may influence metabolism and body composition. Diabetologia 2010, 53:606-613.

23. Vanhoutte $T$, Huys $G$, Brandt E, Swings J: Temporal stability analysis of the microbiota in human feces by denaturing gradient gel electrophoresis using universal and group-specific 16S rRNA gene primers. FEMS Microbiol Ecol 2004, 48:437-446.

24. Kalliomaki M, Collado MC, Salminen S, Isolauri E: Early differences in fecal microbiota composition in children may predict overweight. Am J Clin Nutr 2008, 87:534-538.

25. Zoetendal EG, Rajlic-Stojanovic M, de Vos WM: High-throughput diversity and functionality analysis of the gastrointestinal tract microbiota. Gut 2008, 57:1605-1615.

26. Nagy E, Maier T, Urban E, Terhes G, Kostrzewa M: Species identification of clinical isolates of Bacteroides by matrix-assisted laser-desorption /ionization time-of-flight mass spectrometry. Clin Microbiol Infect 2009, 15:796-802. 
27. Wu X, Ma C, Han L, Nawaz M, Gao F, Zhang X, et al: Molecular characterisation of the faecal microbiota in patients with type II diabetes. Curr Microbiol 2010, 61:69-78.

28. Qin J, Li R, Raes J, Arumugam M, Burgdorf KS, Manichanh C, et al: A human gut microbial gene catalogue established by metagenomic sequencing. Nature 2010, 464:59-65.

29. Fang $\mathrm{H}$, Hedin G: Rapid screening and identification of methicillinresistant Staphylococcus aureus from clinical samples by selective-broth and real-time PCR assay. J Clin Microbiol 2003, 41:2894-2899.

30. Matsuki T, Watanabe K, Fujimoto J, Takada T, Tanaka R: Use of 16S rRNA gene-targeted group-specific primers for real-time PCR analysis of predominant bacteria in human feces. Appl Environ Microbiol 2004, 70:7220-7228.

31. Rinttila T, Kassinen A, Malinen E, Krogius L, Palva A: Development of an extensive set of $16 \mathrm{~S}$ rDNA-targeted primers for quantification of pathogenic and indigenous bacteria in faecal samples by real-time PCR. J Appl Microbiol 2004, 97:1166-1177.

32. Million M, Maraninchi M, Henry M, Armougom F, Richet $H$, Carrieri P, et al: Obesity-associated gut microbiota is enriched in Lactobacillus reuteri and depleted in Bifidobacterium animalis and Methanobrevibacter smithii. Int J Obes (Lond) 2012, 36:817-825.

33. Santacruz A, Marcos A, Warnberg J, Marti A, Martin-Matillas M, Campoy C, et al: Interplay between weight loss and gut microbiota composition in overweight adolescents. Obesity (Silver Spring) 2009, 17:1906-1915.

34. Hartemink R, Domenech VR, Rombouts FM: LAMVAB - A selective medium for the isolation of lactobacilli from faeces. J Microbiol Methods 1997, 29:77-84.

35. Ismail NA, Ragab SH, Elbaky AA, Shoeib ARS, Alhosary Y, Fekry D: Frequency of Firmicutes and Bacteroidetes in gut microbiota of obese and normal weight Egyptian children and adults. Arch Med Sci 2010, 7:501-507.

36. Luoto R, Kalliomaki M, Laitinen K, Isolauri E: The impact of perinatal probiotic intervention on the development of overweight and obesity: follow-up study from birth to 10 years. Int I Obes (Lond) 2010, 34:1531-1537.

37. Cole TJ, Lobstein T: Extended international (IOTF) body mass index cut-offs for thinness, overweight and obesity. Pediatr Obes 2012, 7:284-294.

38. Roelants M, Hauspie R, Hoppenbrouwers K: References for growth and pubertal development from birth to 21 years in Flanders, Belgium. Ann Hum Biol 2009, 36:680-694.

39. Koch FC: Zentralbl. Bakteriol. Parasitenkd. Abt I Orig 1942, 149:122.

40. Murray PR, Rosenthal KS, Kobayashi GS, Pfaller MA: Anaerobic gram-negative bacilli. In Medical Microbiology. St. Louis: Elsevier Mosby; 2002:354-358.

41. Ellner PD, Stoessel CJ, Drakeford E, Vasi F: A new culture medium for medical bacteriology. Tech Bull Regist Med Technol 1966, 36:58-60.

42. Rada V, Petr J: A new selective medium for the isolation of glucose non-fermenting bifidobacteria from hen caeca. J Microbiol Methods 2000 43:127-132.

43. Mariat D, Firmesse O, Levenez F, Guimaraes V, Sokol H, Dore J, et al: The Firmicutes/Bacteroidetes ratio of the human microbiota changes with age. BMC Microbiol 2009, 9:123,

44. Breiman L: Random forests. Mach Learn 2001, 45:5-32

doi:10.1186/1757-4749-5-10

Cite this article as: Bervoets et al:: Differences in gut microbiota composition between obese and lean children: a cross-sectional study. Gut Pathogens 2013 5:10

\section{Submit your next manuscript to BioMed Central and take full advantage of:}

- Convenient online submission

- Thorough peer review

- No space constraints or color figure charges

- Immediate publication on acceptance

- Inclusion in PubMed, CAS, Scopus and Google Scholar

- Research which is freely available for redistribution 